\title{
RESONANCES OF A POTENTIAL WELL WITH A THICK BARRIER
}

\author{
D.C. DOBSON*, F. SANTOSA ${ }^{\dagger}$, S.P. SHIPMAN $\ddagger$, AND M.I. WEINSTEIN $\S$
}

\begin{abstract}
This work is motivated by the desire to develop a method that allows for easy and accurate calculation of complex resonances of a one-dimensional Schrödinger's equation whose potential is a low-energy well surrounded by a thick barrier. The resonance is calculated as a perturbation of the bound state associated with a barrier of infinite thickness. We show that the corrector to the bound state energy is exponentially small in the barrier thickness. A simple computational strategy that exploits this smallness is devised and numerically verified to be very accurate. We also provide a study of high-frequency resonances and show how they can be approximated. Numerical examples are given to illustrate the main ideas in this work.
\end{abstract}

Key words. Resonances for Schrödinger's equation, asymptotic analysis of resonances, approximate methods for calculating resonances

AMS subject classifications. 35Pxx, 24L16, 65L15

1. Introduction. Resonances are important in the study of transient phenomena associated with the wave equation. In particular, long-time behavior of the solution of the wave equation is well described using resonances when radiation losses are small, as in the case of a potential well surrounded by a thick barrier (Fig. 1.1).

Consider the wave equation

$$
\begin{aligned}
& \frac{\partial^{2} u}{\partial t^{2}}=\frac{\partial^{2} u}{\partial x^{2}}-V(x) u \\
& u(x, 0)=u_{0}(x), \quad \frac{\partial u}{\partial t}(x, 0)=u_{1}(x) .
\end{aligned}
$$

Here $V(x)$ is a low-energy potential surrounded by a barrier, and supported in $[-L, L]$. We assume that $u_{0} \in H^{1}([-R, R])$ and $u_{1} \in L^{2}([-R, R])$, where $R>0$. To solve (1.1)-(1.2), we first determine resonances and quasi-modes which are solutions to the nonlinear eigenvalue problem

$$
\begin{aligned}
& -\psi^{\prime \prime}+V(x) \psi=k^{2} \psi, \\
& \psi^{\prime}+i k \psi=0, \text { for } x=L, \\
& \psi^{\prime}-i k \psi=0, \text { for } x=-L .
\end{aligned}
$$

There will be an infinity of resonances $k_{n}$ and their associated quasi-modes $\psi_{n}(x)$. We note that $\operatorname{Im} k_{n}<0$ and assuming that the resonances are simple. Furthermore, for any $A>0$, we can write [13]

$$
u(x, t)=\sum_{\operatorname{Im} k_{n}>-A} c_{j} e^{-i k_{j} t} \psi_{j}(x)+r_{A}(x, t) .
$$

\footnotetext{
*Department of Mathematics, University of Utah, Salt Lake City, UT 84112 (dobson@math.utah.edu).

†School of Mathematics, University of Minnesota, Minneapolis, MN 55455 (santosa@umn.edu).

${ }^{\ddagger}$ Department of Mathematics, Louisiana State University, Baton Rouge, LA 70803 (shipman@math.1su.edu).

$\S$ Appied Physics and Applied Mathematics Department, Columbia University, New York, NY 10027 (miw2103@columbia.edu).
} 


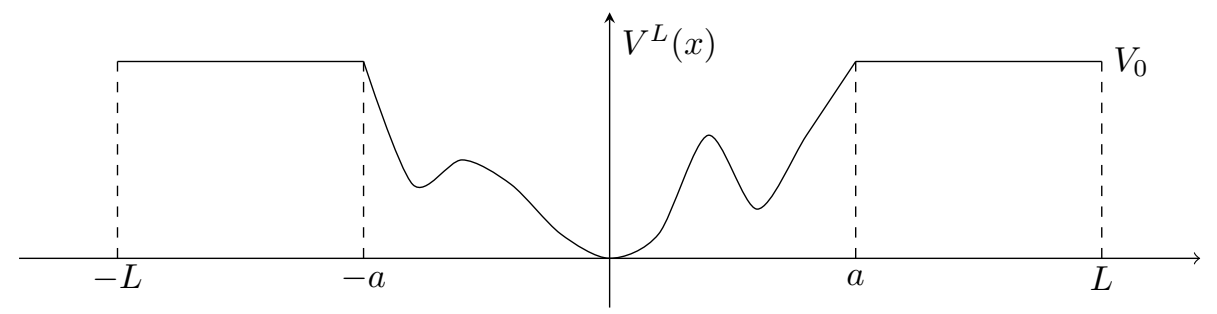

FIG. 1.1. A one-dimensional potential with a well of width a and height $V_{0}$ surrounded by a wall of thickness $(L-a)$. The potential vanishes for $|x|>L$.

The remainder, $r_{A}$, decays in a local energy sense, i.e. for any $K>0$

$$
\left\|r_{A}(t, \cdot)\right\|_{H^{1}([-K, K])} \leq C(R, K) e^{-A t}\left(\left\|u_{0}\right\|_{H^{1}}+\left\|u_{1}\right\|_{L^{2}}\right) .
$$

The importance in the representation (1.6) is that for large $t$, the solution is well described by

$$
u(x, t) \sim c_{j_{0}} e^{-i k_{j_{0}} t} \psi_{j_{0}}(x),
$$

where $j_{0}$ is the index for which $k_{j}$ has the smallest negative imaginary part, i.e. $k_{j_{0}}$ is closest to the real axis. As we shall see below, the size of the negative imaginary part is exponentially small in the barrier thickness $L-a$ (Fig. 1.1).

We refer the reader to Bindel and Zworski $[1,13]$ for a discussion of theoretical, intuitive, and computational aspects of resonances with ample references to the literature. For computational approaches, there are several in literature. Wei, Majda, and Strauss [11] report an algorithm for computing acoustic resonances for a bounded scatterer by relating time samples at a point in space to a quasi-normal mode expansion and using Prony's method to extract the resonant exponents. The method of complex scaling of the spatial variable of Aguilar, Balslev, Combes and Simon [8] can be used to convert resonances into eigenvalues of a modified non-self-adjoint operator - see also Datchev and Zworski [3, 13]. An introduction to this method and its relation to method of perfectly matched layers (PML) can be found in [3]. The potential we consider in this paper can be thought of as a well in an island potential similar to that of Sec. 11 of [5], except that we send the width of the island to infinity.

In this work, we look specifically at computing resonances in certain asymptotic regimes: (1) a potential wall surrounding a well becomes infinitely thick $(L \rightarrow \infty$ with $a$ fixed), keeping $k$ bounded, and (2) $L$ fixed and $\Re k \rightarrow \infty$, high frequencies. As a prototypical example, consider the one-dimensional Schrödinger equation whose potential, $V^{L}(x)$ shown in Figure 1.1. We will assume that $V^{L}(x) \geq 0$ consists of a low-energy well surrounded by a barrier of finite thickness, in this case a wall of height $V_{0}$ and width $(L-a)$. Denote by $V^{\infty}(x)$, the limiting potential well $(L=\infty)$, for which $V^{\infty}(x)=V_{0}$ for $|x|>a$. Introduce

$$
H_{L}=-\partial_{x}^{2}+V^{L}(x) \quad \text { and } \quad H_{\infty}=-\partial_{x}^{2}+V^{\infty}(x) .
$$

For finite $L, H_{L}$ has no bound states. We give a short proof in Appendix A. If $a<L<\infty$, the spectrum of $H_{L}$ is continuous and occupies the nonnegative real line: $\sigma\left(H_{L}\right)=[0, \infty)$. As explained above, a solution with spatially localized initial conditions will decay in the local energy sense as time advances. We are particularly interested in the case where the wall thickness is large and the leakage of energy is 
slow. We shall quantify this leakage rate by relating it to the scattering resonance eigenvalue problem for $V^{L}(x)$.

As $L$ becomes large, two classes of resonances of $H_{L}$ emerge:

(a) A finite number of low-frequency resonances, $E_{\mathrm{res}, i}(L), i=1, \ldots, M$, whose real parts lie in the interval $\left(0, V_{0}\right) \subset \sigma\left(H_{L}\right)$ and which converge, as $L \uparrow$, exponentially fast to the real eigenvalues, $E_{\infty, i}, i=1, \ldots, M$, of $H_{\infty}$ located in the real interval $\left(0, V_{0}\right)$, below the continuous spectrum $\left[V_{0}, \infty\right)$ of $H_{\infty}$. Within the wall in $[-L, L]$, these resonant modes resemble the bound states of $H_{\infty}$, being concentrated in the well in $[-a, a]$; outside of the wall, they grow at a slow exponential rate.

(b) An infinite family of discrete scattering resonances, $E_{\text {c-res }, m}(L), m \geq 1$, which for each fixed $L<\infty$, lies along an unbounded curve in the lower half plane, emanating from the real point $V_{0}$, with $\operatorname{Re} \sqrt{E_{\text {c-res }, m}} \sim \pi \frac{m}{L}$ and $\operatorname{Im} \sqrt{E_{\text {c-res }, m}} \sim-\frac{1}{L} \log \left(\frac{m}{L}\right), m \gg 1$. These discrete points become increasingly clustered along this curve which approaches the continuous spectrum $\left[V_{0}, \infty\right)$, of the limit operator $H_{\infty}$. As $L$ increases, these resonances become increasingly insensitive to the well in $[-a, a]$. See Regge [7], Zworski [12] and Sjöstrand-Zworski [10].

Our main results are as follows:

1. Theorem 2.2 states that for sufficiently large but finite wall thickness $L$, there is in a neighborhood of every discrete eigenvalue of $H_{\infty}$, a nearby scattering resonance in the lower half plane for $H_{L}$. These resonances converge to the eigenvalues of $H_{\infty}$ exponentially fast as $L \rightarrow \infty$. The scattering resonances are fixed points of a complex-valued function whose iterates converge and provide a numerical scheme for computing the resonances.

2. A second approach to the construction and computation of near bound-state scattering resonances is discussed in Section 3. In this approach, the resonances are obtained by viewing $H_{\infty}$ as the unperturbed operator and $H_{L}$ as the perturbed operator. Such a perturbative approach is nontrivial, since $V^{L}(x)-V^{\infty}(x)=-V_{0} \mathbf{1}_{[L, \infty)}(x)$ is a non-compact perturbation of $V^{\infty}$; while the eigenvalues of $V^{\infty}(x)$ are real with $L^{2}$ eigenfunctions, those for $H_{L}$ are complex non- $L^{2}$ (only $L_{\text {loc }}^{2}$ ) eigenstates. We numerically implement this perturbative approach and demonstrate, for $L$ large, that it gives excellent agreement with very accurate direct numerical calculations of the resonances of $H_{L}$.

3. For computing high-frequency resonances, we offer in Section 4 an approximate iterative numerical scheme based on leading terms of the high- $E$ asymptotics of solutions of the ODE. The scheme excludes terms that depend on the particular form of the well. In two simple cases, $V(x)=V_{0}$ (no well) and $V(x)=0$ (square well) in $|x|<a$, we are able to compare the results of the scheme with the exact values of the resonances. In the latter case and for small $L$, there is appreciable imprecision in the imaginary part of the computed resonances. This error vanishes as $L$ increases, indicating that the shape of the well has a negligible effect as, $L$, the width of the wall increases.

2. Near bound-state resonances. Assuming $E>0$, let us put $E=k^{2}$, with Re $k>0$. For simplicity, we consider a symmetric potential $V(-x)=V(x)$. In this case all quasi-normal modes are even or odd. We restrict our attention to even quasi-normal modes (scattering resonances) for which the pair $(\psi(x), k)$ satisfies the 
eigenvalue problem:

$$
\begin{aligned}
& H_{L} \psi:=-\psi^{\prime \prime}+V^{L}(x) \psi=k^{2} \psi, \quad 0<x<\infty, \\
& \quad \psi(0)=1, \quad \psi^{\prime}(0)=0 \\
& \psi^{\prime}(L)-i k \psi(L)=0
\end{aligned}
$$

in which the prime indicates differentiation with respect to $x$. The potential is of the type illustrated in Figure 1.1:

$$
V^{L}(x)=\left\{\begin{array}{ll}
v(x) & \text { for } \quad 0 \leq x<a \\
V_{0} & \text { for } \quad a \leq x<L \\
0 & \text { for } \quad x \geq L
\end{array},\right.
$$

where $0 \leq v(x) \leq V_{0}$. The initial conditions (2.2) imply that $\psi(x)$ can be continued to a symmetric function on $\mathbb{R}$ satisfying outgoing radiation conditions at $\pm \infty$. Condition (2.3) is the outgoing radiation condition. Due to the boundary condition at $x=L$, the boundary-value problem (2.1)-(2.3) is non-self-adjoint. Thus, its eigenvalues can be expected to be complex and, in fact, form a discrete subset of the open lower half plane; see Appendix B.

2.1. The resonance condition. We next derive a characterization of scattering resonance energies, $k$, as zeros of a function defined on a subset of $\mathbb{C}$. Denote by $\psi(x, k)$ the unique solution of the initial value problem (2.1)-(2.2). For $V(x) \equiv V_{0}$, for $a \leq x \leq L$, we will also be able to make use the explicit form of the solution in this region,

$$
\psi(x)=A e^{i \sqrt{k^{2}-V_{0}}(x-a)}+B e^{-i \sqrt{k^{2}-V_{0}}(x-a)},
$$

where $A, B$ are unknown constants. For $x>L, V(x)=0$, and applying the radiation boundary condition (2.3), we obtain

$$
\psi(x)=C e^{i k(x-L)}, \quad x>L,
$$

where $C$ is also unknown.

We first briefly deal with the case $L=\infty$. The condition for $H_{\infty}$ that is consistent with the outgoing condition in the case $L<\infty$, is that $\psi^{\prime}(a)-i \sqrt{k^{2}-V_{0}} \psi(a)=0$, or

$$
\psi(x)=A e^{i \sqrt{k^{2}-V_{0}}(x-a)}, \quad x>a .
$$

For $k^{2}<V_{0}$, this condition implies decay of the solution. Since $\psi(a, k) \neq 0$ the condition for a bound state can be written as

$$
\sqrt{k^{2}-V_{0}}-\frac{\psi^{\prime}(a, k)}{i \psi(a, k)}=0 .
$$

Equation (2.7) has a finite number of solutions (a proof is given in Appendix C) giving rise to eigenvalues of $H_{\infty}$ :

$$
E_{\infty, i}=k_{\infty, i}^{2} \in\left(0, V_{0}\right), \quad i=1, \ldots, M
$$

These eigenvalues are simple, corresponding to the zeros, $k_{B}$, of the expression in (2.7) being simple. We present a proof of this in Appendix D. For the remainder we will denote by $k_{B}$ a generic bound state frequency satisfying (2.7). 
For $L$ finite, we now derive the extension of (2.7) to resonances. Continuity of $\psi$ and $\psi^{\prime}$ at $x=a$ and (2.5) imply

$$
\psi(a)=A+B, \text { and } \psi^{\prime}(a)=i \sqrt{k^{2}-V_{0}}(A-B) .
$$

Continuity of $\psi$ and $\psi^{\prime}$ at $x=L$ implies

$$
\begin{aligned}
A e^{i \sqrt{k^{2}-V_{0}}(L-a)}+B e^{-i \sqrt{k^{2}-V_{0}}(L-a)} & =C, \\
i \sqrt{k^{2}-V_{0}}\left(A e^{i \sqrt{k^{2}-V_{0}}(L-a)}-B e^{-i \sqrt{k^{2}-V_{0}}(L-a)}\right) & =i k C .
\end{aligned}
$$

Eliminating $A, B$, and $C$, we arrive at an equation for the resonance energies $E=k^{2}$ :

$$
\begin{aligned}
& \left(\psi(a, k)+\frac{\psi^{\prime}(a, k)}{i \sqrt{k^{2}-V_{0}}}\right)\left(1-\frac{\sqrt{k^{2}-V_{0}}}{k}\right) e^{i \sqrt{k^{2}-V_{0}}(L-a)} \\
& +\left(\psi(a, k)-\frac{\psi^{\prime}(a, k)}{i \sqrt{k^{2}-V_{0}}}\right)\left(1+\frac{\sqrt{k^{2}-V_{0}}}{k}\right) e^{-i \sqrt{k^{2}-V_{0}}(L-a)}=0 .
\end{aligned}
$$

Multiplication of (2.9) by

$$
\frac{\left(k \sqrt{k^{2}-V_{0}}\right) e^{i \sqrt{k^{2}-V_{0}}(L-a)}}{\psi(a, k)\left(k+\sqrt{k^{2}-V_{0}}\right)}
$$

yields the equivalent equation

$$
F_{B}(k)+g(k) e^{2 i \sqrt{k^{2}-V_{0}}(L-a)}=0, \quad \text { (resonance condition) }
$$

where

$$
\begin{aligned}
& F_{B}(k) \equiv \sqrt{k^{2}-V_{0}}-\frac{\psi^{\prime}(a, k)}{i \psi(a, k)}, \\
& g(k) \equiv\left(\sqrt{k^{2}-V_{0}}+\frac{\psi^{\prime}(a, k)}{i \psi(a, k)}\right) \frac{k-\sqrt{k^{2}-V_{0}}}{k\left(k+\sqrt{k^{2}-V_{0}}\right)} .
\end{aligned}
$$

By (2.7), the bound state energies $k_{B}^{2}$ of $H_{\infty}$ are characterized by $F_{B}\left(k_{B}\right)=0$, and $0<k_{B}^{2}<V_{0}$. The scattering resonance energies are solutions of the perturbed equation (2.10).

Remark 2.1 Equation (2.10) for the scattering resonance energies is equivalent to

$$
\mathcal{W}(k ; L) \equiv \phi_{1}(x, k) \partial_{x} \phi_{2}(x ; k)-\phi_{2}(x ; k) \partial_{x} \phi_{1}(x ; k)=0,
$$

where $\mathcal{W}(k ; L)$ is the Wronskian of the two solutions $\phi_{j}(x, k), j=1,2$, satisfying $H_{L} \phi=k^{2} \phi$ with $\phi_{1}$ satisfying the boundary condition (2.2), and $\phi_{2}$ satisfying the boundary condition $(2.3)$ and $\phi_{2}(L ; k)=1$. The Green function for $-\partial_{x}^{2}+V-k^{2}$ explicitly given by variation of constants, has poles (scattering resonances) in $\operatorname{Im} k<0$, at the zeros of $\mathcal{W}(k ; L)$. 
2.2. Resonances converging to eigenvalues as $L \rightarrow \infty$. We now construct the complex scattering resonances for $H_{L}$ and prove that they converge exponentially to the discrete eigenvalues $\left\{k_{B}\right\}$ of $H_{\infty}$ as $L \rightarrow \infty$.

Write $k=k_{B}+\kappa$, recalling that $k_{B}$ is real, and anticipate that $\kappa$ is complex. From (2.10) we have

$$
F_{B}\left(k_{B}+\kappa\right)+g\left(k_{B}+\kappa\right) \exp \left\{-2 \sqrt{V_{0}-\left(k_{B}+\kappa\right)^{2}}(L-a)\right\}=0 .
$$

We shall seek solutions to (2.13) in the complex $\kappa$ plane. These turn out to lie in the lower half plane and, for $L$ large, exponentially close to the real zeros of $F_{B}(k)$.

Since $F_{B}\left(k_{B}\right)=0$, and $k_{B}$ is a simple zero of $F_{B}$, we have

$$
F_{B}\left(k_{B}+\kappa\right)=\kappa \int_{0}^{1} F_{B}^{\prime}\left(k_{B}+\theta \kappa\right) d \theta \equiv \kappa f_{1}\left(k_{B}+\kappa\right) .
$$

$F_{B}^{\prime}\left(k_{B}\right) \neq 0$ and $f_{1}\left(k_{B}+\kappa\right)$ is non-vanishing for $|\kappa|$ sufficiently small. Thus (2.13), may be rewritten as

$$
\kappa=G(\kappa):=-\frac{g\left(k_{B}+\kappa\right)}{f_{1}\left(k_{B}+\kappa\right)} \exp \left\{-2 \sqrt{V_{0}-\left(k_{B}+\kappa\right)^{2}}(L-a)\right\} .
$$

Theorem 2.2 Let $k_{B}$ be such that $F_{B}\left(k_{B}\right)=0$, and thus $0<k_{B}^{2}<V_{0}$ is a bound state energy of $H_{\infty}$.

1. There are positive constants $C, \alpha$, and $L^{\prime}$ such that, if $L>L^{\prime}$, then the scattering resonance eigenvalue problem (2.1-2.3) has a solution with scattering resonance eigenvalue $k=k_{B}+\kappa$ and

$$
|\kappa| \leq C e^{-2 \alpha(L-a)} .
$$

2. $\operatorname{Im} \kappa<0$ and

$$
\kappa \sim K e^{-2 \sqrt{V_{0}-k_{B}^{2}} L} \quad(L \rightarrow \infty)
$$

with $K=-g\left(k_{B}\right) f_{1}\left(k_{B}\right)^{-1} \exp \left(2 a \sqrt{V_{0}-k_{B}^{2}}\right)$, in the sense that the ratio of the two sides of the expression tends to 1.

Proof. To prove the theorem we use the following claim, proved below:

Claim: $f_{1}\left(k_{B}+\kappa\right)$ and $g\left(k_{B}+\kappa\right)$ are analytic in a neighborhood of $\kappa=0$ and there exist positive constants $C, \alpha$, and $r$ such that

$$
\left|\frac{g\left(k_{B}+\kappa\right)}{f_{1}\left(k_{B}+\kappa\right)}\right| \leq C \text { and } \operatorname{Re} \sqrt{V_{0}-\left(k_{B}+\kappa\right)^{2}} \geq \alpha, \text { for }|\kappa|<r .
$$

The theorem then is proved as follows. Let $L^{\prime}$ be such that, for $L \geq L^{\prime}$,

$$
C e^{-2 \alpha(L-a)}<r \text { and }\left|\frac{d G(\kappa)}{d \kappa}\right| \leq 0.99, \text { for }|\kappa|<r .
$$

The bound on $\partial G / \partial \kappa$ comes from the analyticity of $g / f_{1}$ and the exponential factor in (2.15). It follows that for $|\kappa|<r$, we have

$$
|G(\kappa)| \leq C e^{-2 \alpha(L-a)}<r .
$$


Thus $G$ maps the disk $\{|\kappa|<r\}$ into itself. Furthermore, $G$ is a contraction on this disk because $\left|G^{\prime}(\kappa)\right|<1$ for $|\kappa|<r$. It follows that (2.15) has a unique solution in $\{|\kappa|<r\}$, which satisfies the bound (2.18). Therefore, to complete the proof, we need only verify the above claim.

To verify the claim it suffices to show that $f_{1}\left(k_{B}+\kappa\right)$ does not vanish in some neighborhood $k_{B}$. The expression for $f_{1}$ in $(2.14)$ suggests calculation of $F_{B}^{\prime}\left(k_{B}\right)$, using that $F\left(k_{B}\right)=0$. We obtain

$$
\begin{aligned}
& \left.\frac{d}{d k} F_{B}(k)\right|_{k=k_{B}}=\left.\frac{d}{d k}\left(k+\sqrt{k^{2}-V_{0}}\right) \cdot\left(\sqrt{k^{2}-V_{0}}-\frac{\psi^{\prime}(a, k)}{i \psi(a, k)}\right)\right|_{k=k_{B}} \\
& +\left.\left(k_{B}+\sqrt{k_{B}^{2}-V_{0}}\right) \cdot \frac{d}{d k}\left(\sqrt{k^{2}-V_{0}}-\frac{\psi^{\prime}(a, k)}{i \psi(a, k)}\right)\right|_{k=k_{B}} \\
& =\left.\left(k_{B}+\sqrt{k_{B}^{2}-V_{0}}\right) \cdot \frac{d}{d k}\left(\sqrt{k^{2}-V_{0}}-\frac{\psi^{\prime}(a, k)}{i \psi(a, k)}\right)\right|_{k=k_{B}}
\end{aligned}
$$

We now observe that $F_{B}^{\prime}\left(k_{B}\right) \neq 0$. Indeed, the second factor in $(2.19)$, is the derivative at $k=k_{B}$ of the function detecting bound states of $H_{\infty}$, which is nonzero (Appendix D). Thus, $F_{B}^{\prime}\left(k_{B}\right) \neq 0$ and by analyticity of $F_{B}(k)$ near $k_{B}$ and the expression for $f_{1}$ in (2.14), there exists $r>0$, such that

$$
\left|f_{1}\left(k_{B}+\kappa\right)\right|>0 \text { for }|\kappa|<r .
$$

Finally, let $\kappa=\kappa_{1}+i \kappa_{2}$ and consider

$$
V_{0}-\left(k_{B}+\kappa\right)^{2}=\left(V_{0}-k_{B}^{2}-2 k_{B} \kappa_{2}-\kappa_{1}^{2}+\kappa_{2}^{2}\right)-2 i\left(k_{B} \kappa_{2}+\kappa_{1} \kappa_{2}\right) .
$$

Since $V_{0}>k_{B}^{2}$, there by perhaps taking $r$ smaller, but still positive, we have that for $|\kappa|<r$, the real part of $\left(V_{0}-\left(k_{B}+\kappa\right)^{2}\right)>0$, and hence

$$
\operatorname{Re} \sqrt{V_{0}-\left(k_{B}+\kappa\right)^{2}} \geq \alpha>0
$$

for some $\alpha$. This completes the proof of the claim and therewith Part 1 of Theorem 2.2. Part 2 comes from (2.15), the fact that $\kappa \rightarrow 0$ exponentially as $L \rightarrow \infty$, and the fact that $g\left(k_{B}\right)$ and $f_{1}\left(k_{B}\right)$ are nonzero. That $\operatorname{Im} \kappa<0$ is standard; see Appendix B.

Remark 2.3 This result shows that for a resonance $k$ near a bound state $k_{B}, k-k_{B}$ is exponentially small in $(L-a)$, or the thickness of the wall. The equation (2.15) suggests that for $L-a \gg 1$

$$
\kappa \approx \kappa_{1}=-\frac{g\left(k_{B}\right)}{F_{B}^{\prime}\left(k_{B}\right)} \exp \left\{-2 \sqrt{V_{0}-\left(k_{B}\right)^{2}}(L-a)\right\} .
$$

2.3. A numerical illustration. To illustrate the conclusions of Theorem 2.2 we consider the simple example of a piecewise constant potential:

$$
V_{L}(x)=\left\{\begin{array}{lll}
0 & \text { for } & 0 \leq x<a \\
V_{0} & \text { for } & a \leq x<L \\
0 & \text { for } & x \geq L
\end{array} .\right.
$$

For this case,

$$
\psi(x, k)=\cos k x, \text { for } 0 \leq x<a .
$$




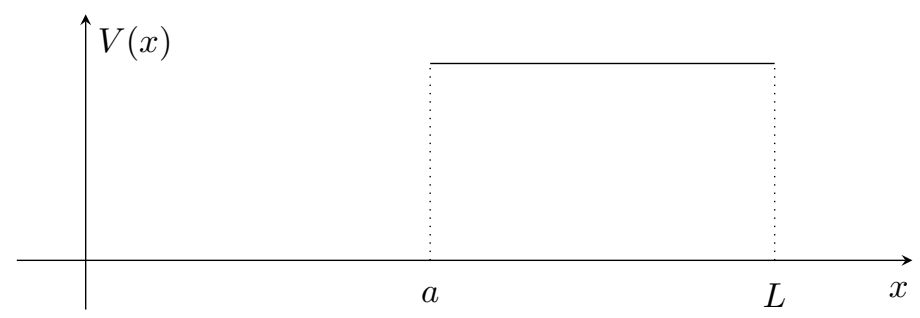

FIG. 2.1. The potential used in the example in $\S 3$.

The equation for the bound states (2.7) simplifies to

$$
-k \tan k a=i \sqrt{k^{2}-V_{0}}
$$

which has solution(s) for $k<\sqrt{V_{0}}$. We denote the bound state $k$ by $k_{B}$. The equation for resonances (2.9) becomes

$$
\begin{aligned}
& \left(\cos k a-\frac{k \sin k x}{i \sqrt{k^{2}-V_{0}}}\right)\left(1-\frac{\sqrt{k^{2}-V_{0}}}{k}\right) e^{i \sqrt{k^{2}-V_{0}}(L-a)} \\
& +\left(\cos k a+\frac{k \sin k a}{i \sqrt{k^{2}-V_{0}}}\right)\left(1+\frac{\sqrt{k^{2}-V_{0}}}{k}\right) e^{-i \sqrt{k^{2}-V_{0}}(L-a)}=0 .
\end{aligned}
$$

Let us denote the resonances by $k=k_{B}+\kappa$, which are complex numbers with negative imaginary parts.

As an example, we set $V_{0}=4$ and $a=1$. We then use Newton's method to solve for $k_{B}$ and $k$ for different barrier widths $L$. There are a finite number of solutions $k_{B}$ of $F_{B}(k)=0$. The lowest energy bound state is $k_{B}=1.02986652932226$. The table below shows the dependence of the resonance $k$, which is a perturbation of $k_{B}$, approaching $k_{B}$ as $L \uparrow \infty$ by Theorem 2.2. Note the exponential decay of $\kappa$ in $L$.

\begin{tabular}{|c|c|}
\hline$L$ & $k$ \\
\hline \hline 3 & $1.02959049042796-0.00051708051625 i$ \\
\hline 4 & $1.02985760250922-0.00001677632024 i$ \\
\hline 5 & $1.02986623992970-0.00000054394364 i$ \\
\hline 6 & $1.02986651993964-0.00000001763573 i$ \\
\hline
\end{tabular}

The dependence of a resonance on the barrier thickness $L$. The barrier height is $V_{0}=4$ and and the defect thickness is $a=1$.

Table 2.2 shows the real and imaginary parts of $\kappa=k-k_{B}$. It is clear from the table that $\kappa$ decays exponentially as a function of $(L-a)$. These results illustrate the estimate in Theorem 2.2. They also illustrate that, even for barriers that are not so thick $(L=3)$, the estimate of $k_{B}$ is very good-the exponential decay of $\kappa$ provides very quick convergence.

3. Direct perturbation for near bound-state resonances. We now take a direct approach to the construction of near-bound states as perturbations of true bound states. This leads to a computational method, explored in sections 3.2-3.3. We remark that parts of this approach may be applicable to problems in higher dimensions. 


\begin{tabular}{|c|c|c|}
\hline$L-a$ & $\operatorname{Re} \kappa$ & $\operatorname{Im} \kappa$ \\
\hline \hline 2 & $-2.76038894295727 \times 10^{-4}$ & $-5.17080516251752 \times 10^{-4}$ \\
\hline 3 & $-8.92681303588105 \times 10^{-6}$ & $-1.67763202402731 \times 10^{-5}$ \\
\hline 4 & $-2.89392557917267 \times 10^{-7}$ & $-5.43943636731303 \times 10^{-7}$ \\
\hline 5 & $-9.38261446314925 \times 10^{-9}$ & $-1.76357251517556 \times 10^{-9}$ \\
\hline
\end{tabular}

The real and imaginary parts of $\kappa$ for different $L$. Note the negative exponential dependence on $(L-a)$. Here, $V_{0}=4$ and $a=1$.

3.1. Direct perturbation of bound states. For a compactly supported potential $V^{L}$, it is possible to reduce the scattering resonance problem (2.1)-(2.3) to a nonlocal (boundary integral) equation at $x=L$. We seek solutions of (2.1)-(2.3) in the form

$$
\psi(x ; k)=\psi_{B}(x)+\xi(x), \quad k=k_{B}+\kappa,
$$

where

$$
H_{\infty} \psi_{B}=k_{B}^{2} \psi_{B}, \quad \psi_{B} \in L^{2}(\mathbb{R}), \quad 0<k_{B}^{2}<V_{0} .
$$

Substitution of (3.1) into (2.1)-(2.3) yields the following system for $\xi(x)$ and $\kappa$ :

$$
\begin{aligned}
& -\xi^{\prime \prime}+V^{\infty}(x) \xi-\left(k_{B}+\kappa\right)^{2} \xi=\left(2 k_{B} \kappa+\kappa^{2}\right) \psi_{B}, \quad 0<x<L, \\
& \xi(0)=0, \quad \xi^{\prime}(0)=0, \\
& \xi^{\prime}-i\left(k_{B}+\kappa\right) \xi=-\psi_{B}^{\prime}+i\left(k_{B}+\kappa\right) \psi_{B}, \quad x=L .
\end{aligned}
$$

In obtaining (3.2) we used that $V^{L}(x)=V^{\infty}(x)$ for $0 \leq x \leq L$. One must determine $\kappa$ such that (3.4) is satisfied.

Introduce $f \mapsto S_{k} f$ the solution operator of the inhomogeneous initial value problem:

$$
\begin{aligned}
-u^{\prime \prime}+V^{\infty}(x) u-k^{2} u & =f, \quad 0<x<L, \\
u(0)=0, \quad u^{\prime}(0) & =0 .
\end{aligned}
$$

Note that for each fixed $f \in C^{0}([0, L])$, the mapping $k \mapsto S[k] f$ varies smoothly with $k$. Equation (3.2)-(3.3) can now be solved in terms of the operator $S_{k_{B}+\kappa}$ :

$$
\xi=\left(2 k_{B} \kappa+\kappa^{2}\right) S_{k_{B}+\kappa} \psi_{B}
$$

Substitution of (3.5) into the outgoing radiation condition (3.4) yields a nonlocal equation for $\kappa=\kappa(L)$, the correction to $k_{B}$, due to finite barrier width. Anticipating that $\kappa$ will be small, we organize the terms of this equation in ascending order in $\kappa$ :

$$
\begin{aligned}
& \psi_{B}^{\prime}-i k_{B} \psi_{B}+\kappa q_{B}(L)+ \\
& +\kappa\left[2 k_{B}\left(\partial-i k_{B}\right)\left\{S_{k_{B}+\kappa}-S_{k_{B}}\right\} \psi_{B}\right] \\
& +\kappa^{2}\left[\partial S_{k_{B}+\kappa} \psi_{B}-i\left(3 k_{B}+\kappa\right) S_{k_{B}+\kappa} \psi_{B}\right]=0,
\end{aligned}
$$

in which

$$
q_{B}(L) \equiv\left[2 k_{B}\left(\partial-i k_{B}\right) S_{k_{B}} \psi_{B}-i \psi_{B}\right] .
$$


In (3.6), we have used the following conventions: $\partial S_{k} f=\left.\partial_{x}\left(S_{k} f\right)\right|_{x=L}$ and all evaluations of $\psi_{B}, S_{k_{B}} \psi_{B}$, and $\partial S_{k_{B}} \psi_{B}$ are at $x=L$ and $k=k_{B}$. By smoothness of $k \mapsto S_{k} f$, the terms on the second and third lines of (3.6) can be expressed, for $\kappa$ in a neighborhood of zero, in the form $-\kappa \Lambda(\kappa, L)$, where $\Lambda$ is smooth in $\kappa$.

Thus we can re-express (3.6) as

$$
q_{B}(L) \kappa=i k_{B} \psi_{B}(L)-\psi_{B}^{\prime}(L)-\kappa \Lambda(\kappa, L)
$$

or

$$
\kappa=\frac{i k_{B} \psi_{B}(L)-\psi_{B}^{\prime}(L)}{q_{B}(L)}-\kappa \frac{\Lambda(\kappa, L)}{q_{B}(L)},
$$

where

$$
\begin{aligned}
\Lambda(\kappa, L)= & {\left[2 k_{B}\left(\partial-i k_{B}\right)\left(S_{k_{B}+\kappa} \psi_{B}-S_{k_{B}} \psi_{B}\right)\right] } \\
& +\kappa\left[\partial S_{k_{B}+\kappa} \psi_{B}-i\left(3 k_{B}-\kappa\right) S_{k_{B}+\kappa} \psi_{B}\right]
\end{aligned}
$$

We will show that (3.8) admits a solution and that it is exponentially small in $L$.

Notice that $V^{L}(x)=V_{0}$ for $x>a$. Therefore, from (3.1) we have

$$
\psi_{B}(x)=\psi_{B}(a) e^{-\sigma_{B}(x-a)}, \quad x>a,
$$

where $\sigma_{B}=\sqrt{V_{0}-k_{B}^{2}}>0$. To study the properties of $q_{B}(L)$, consider $u_{B}(x)$ satisfying

$$
-u_{B}^{\prime \prime}+\sigma_{B}^{2} u_{B}=\psi_{B}(a) e^{-\sigma_{B}(x-a)}, \quad x>a .
$$

This can be solved explicitly to give

$$
u_{B}(x)=C_{1} e^{\sigma_{B}(x-a)}+C_{2} e^{-\sigma_{B}(x-a)}+\frac{\psi_{B}(a)}{2 \sigma_{B}} x e^{-\sigma_{B}(x-a)}, \quad x>a .
$$

Observe that for $x>a, u_{B}(x)=S_{k_{B}} \psi_{B}$ for some choice of $C_{1}$ and $C_{2}$. From (3.7), it is clear that $q_{B}(L)$ is dominated by the positive exponential terms in $u_{B}(x)$ as long as $C_{1} \neq 0$. Therefore, we argue that, for some $L_{0}>0$ and $C>0$,

$$
\left|q_{B}(L)\right| \geq C e^{\sigma_{B} L},
$$

for all $L>L_{0}$. We show that $C_{1} \neq 0$ in Appendix D.

Theorem 3.1 For some $r>0$ (3.8) admits a solution $\kappa$ with $|\kappa|<r$. Moreover, $\kappa$ is exponentially small in $L$ for sufficiently large $L$.

Proof. We examine the iterative approach to solve (3.8). We write

$$
\begin{aligned}
\kappa_{0} & =\frac{i k_{B} \psi_{B}(L)-\psi_{B}^{\prime}(L)}{q_{B}(L)}, \\
\kappa_{n+1} & =\kappa_{0}-\kappa_{n} \frac{\Lambda\left(\kappa_{n}, L\right)}{q_{B}(L)} .
\end{aligned}
$$

Our aim is to show that

$$
\left|\kappa_{n+1}-\kappa_{n}\right| \leq C_{0}\left|\kappa_{n}-\kappa_{n-1}\right|,
$$


for some $C_{0}<1$, and that the iterates remain within some small ball centered at the origin.

Since $L>a$, we can use (3.1) to obtain

$$
\kappa_{0}=\frac{\left(i k_{B}-\sigma_{B}\right) \psi_{B}(a) e^{-\sigma_{B}(L-a)}}{q_{B}(L)} .
$$

This, together with (3.10) leads to

$$
\left|\kappa_{0}\right| \leq C_{0} e^{-2 \sigma_{B} L},
$$

for some $C_{0}$ independent of $L$. From (3.12), we have

$$
\left|\kappa_{n+1}\right| \leq\left|\kappa_{0}\right|+\left|\kappa_{n}\right| \frac{\left|\Lambda\left(\kappa_{n}, L\right)\right|}{\left|q_{B}(L)\right|}
$$

Suppose

$$
\left|\kappa_{n}\right| \leq 2 C_{0} e^{-2 \sigma_{B} L}
$$

then

$$
\left|\kappa_{n+1}\right| \leq C_{0} e^{-2 \sigma_{B} L}\left(1+2 \frac{\left|\Lambda\left(\kappa_{n}, L\right)\right|}{\left|q_{B}(L)\right|}\right),
$$

and we must show that $\left|\Lambda\left(\kappa_{n}, L\right)\right| /\left|q_{B}(L)\right|<1 / 2$ for sufficiently large $L$.

In the definition of $\Lambda(\kappa, L)(3.9)$, there are two terms. To obtain a bound for the second term, we need to consider $S_{k_{B}+\kappa} \psi_{B}$. Let $u(x)$ satisfy

$$
-u^{\prime \prime}+\sigma^{2} u=\psi_{B}(a) e^{-\sigma_{B}(x-a)}, \quad x>a,
$$

where $\sigma=\sqrt{V_{0}-k^{2}}$ and $k=k_{B}+\kappa_{n}$. Then $u(x)=S_{k} \psi_{B}$ and is given by

$$
u(x)=C_{1} e^{\sigma(x-a)}+C_{2} e^{-\sigma(x-a)}+\frac{\psi_{B}(a) e^{-\sigma_{B}(x-a)}}{\sigma^{2}-\sigma_{B}^{2}}, x>a .
$$

One can evaluate $C_{1}$ and $C_{2}$ using boundary conditions $u(a)$ and $u^{\prime}(a)$. In doing so, one can see that the expression approaches $\left[S_{k_{B}} \psi_{B}\right](x)$ as $\sigma \rightarrow \sigma_{B}$.

For $k=k_{B}+\kappa$, we have

$$
\sigma=\sqrt{V_{0}-\left(k_{B}+\kappa\right)^{2}}=\sigma_{B} \sqrt{1-\frac{2 k_{B} \kappa-\kappa^{2}}{\sigma_{B}^{2}}} .
$$

Suppose $|\kappa| \leq 2 C_{0} e^{-2 \sigma L}$ as in (3.13), then we can conclude that

$$
\operatorname{Re} \sigma>0, \quad \text { and } \quad|\sigma| \leq \sigma_{B}(1+s|\kappa|) \text { for }|\kappa|<r
$$

for some $s>0$. It is clear then that $u(x)$ is dominated by the positive exponential term for large $x$ and therefore

$$
\left|\left[S_{k_{B}+\kappa} \psi_{B}\right](L)\right|=|u(L)| \leq C_{3} e^{\sigma_{B} L} e^{s|\kappa| L} .
$$

Similarly, we argue that

$$
\left|\left[S_{k_{B}+\kappa} \psi_{B}\right](L)\right|=\left|u^{\prime}(L)\right| \leq C_{4} e^{\sigma_{B} L} e^{s|\kappa| L} .
$$


To study the first term of $\Lambda(\kappa, L)$, we need to consider $S_{k_{B}+\kappa} \psi_{B}-S_{k_{B}} \psi_{B}$. Since $S_{k} \psi_{B}$ is a smooth function of $k$, we can use the fundamental theorem of calculus and write

$$
S_{k_{B}+\kappa} \psi_{B}-S_{k_{B}} \psi_{B}=\int_{0}^{1} \kappa \partial_{k} S_{k_{B}+\tau \kappa} \psi_{B} d \tau
$$

Let $\dot{u}(x)$ satisfy the $k$-differentiated version of (3.15), i.e.,

$$
-\dot{u}^{\prime \prime}+\sigma^{2} \dot{u}=2 k u, \quad x>a .
$$

Then $\dot{u}(x)=\partial_{k} S_{k} \psi_{B}$, and is given by

$$
\begin{aligned}
\dot{u}(x) & =D_{1} e^{\sigma(x-a)}+D_{2} e^{-\sigma(x-a)} \\
& -\frac{k x}{\sigma} C_{1} e^{\sigma(x-a)}+\frac{k x}{\sigma} C_{2} e^{-\sigma(x-a)}+\frac{2 k \psi_{B}(a) e^{-\sigma_{B}(x-a)}}{\sigma^{2}-\sigma_{B}^{2}} .
\end{aligned}
$$

One concludes that if $C_{1}$ does not vanish,

$$
|\dot{u}(L)| \leq C_{5} e^{\sigma_{B} L} e^{s|\kappa| L} .
$$

The estimates for $u(L), u^{\prime}(L)$, and $\dot{u}(L)$ lead to the following estimate for $\Lambda(\kappa, L)$

$$
|\Lambda(\kappa, L)| \leq \kappa C_{6} e^{\sigma L} e^{s|\kappa| L} .
$$

Considering the bound in (3.14) and using (3.10), we arrive at

$$
\frac{\left|\Lambda\left(\kappa_{n}, L\right)\right|}{\left|q_{B}(L)\right|} \leq C \exp \left[-L\left(2 \sigma_{B}+2 s C_{0} e^{-2 \sigma_{B} L}\right)\right]
$$

It is apparent that we can choose $L$ sufficiently large so that the expression above is less than $1 / 2$. Therefore, the iterates (3.12) starts and stays in an exponentially small (in $L$ ) ball.

To show that the iterates converge, we rewrite

$$
\begin{aligned}
\kappa_{n+1}-\kappa_{n} & =-\kappa_{n} \frac{\Lambda\left(\kappa_{n}, L\right)}{q_{B}(L)}+\kappa_{n-1} \frac{\Lambda\left(\kappa_{n-1}, L\right)}{q_{B}(L)} \\
& =-\left(\kappa_{n}-\kappa_{n-1}\right) \frac{\Lambda\left(\kappa_{n}, L\right)}{q_{B}(L)}-\kappa_{n-1} \frac{\Lambda\left(\kappa_{n}, L\right)-\Lambda\left(\kappa_{n-1}, L\right)}{q_{B}(L)} .
\end{aligned}
$$

From the above bound on $\left|\Lambda\left(\kappa_{n}, L\right) /\right| q_{B}(L) \mid$, we obtain

$$
\left|\kappa_{n+1}-\kappa_{n}\right| \leq\left|\kappa_{n}-\kappa_{n-1}\right|\left(\frac{1}{2}+2 C_{o} e^{-2 \sigma_{B} L} \frac{\left|\Lambda\left(\kappa_{n}, L\right)-\Lambda\left(\kappa_{n-1}, L\right)\right|}{q_{B}(L)\left|\kappa_{n}-\kappa_{n-1}\right|}\right) .
$$

Convergence is guaranteed as long as $\left|\Lambda\left(\kappa_{n}, L\right)-\Lambda\left(\kappa_{n-1}, L\right)\right| /\left(\left|q_{B}(L)\right|\left|\kappa_{n}-\kappa_{n-1}\right|\right)$ is bounded.

We omit the demonstration of such a bound since the method is very similar to that used to establish the (3.17), namely, the explicit use of the solution of the constant coefficient differential equation for $x>a$. This completes the proof. 
3.2. A computational framework for near bound-state resonances. The perturbative approach of section 3 suggests a computational approach to near bound state resonances, based on the (3.8) and the method of successive approximations.

Step 1. Solve for a bound state eigenpair $\left(\psi_{B}, E_{B}=k_{B}^{2}\right)$ of $H^{\infty}$ in (3.1), corresponding to the limiting potential.

Step 2. Solve the initial value problem

$$
\begin{aligned}
-u_{B}^{\prime \prime}+V^{\infty}(x) u_{B}-k_{B}^{2} u_{B} & =\psi_{B}(x), \quad 0<x<L \\
u_{B}(0)=1, \quad u_{B}^{\prime}(0) & =0
\end{aligned}
$$

for the unique solution, and denote it by $S_{k_{B}} \psi_{B}(x)$.

Step 3: Define the correction $\kappa_{0}$, based on the leading order term in (3.8) by:

$$
\kappa_{0} \equiv \frac{\left(i k_{B} \psi_{B}(L)-\psi_{B}^{\prime}(L)\right)}{\left.2 k_{B}\left(\partial-i k_{B}\right) S_{k_{B}} \psi_{B}\right|_{x=L}-i \psi_{B}(L)} .
$$

Step 4. Approximate the resonance eigenpair with energy $k$ near $k_{B}$ by

$$
\begin{aligned}
k_{\text {approx }} & =k_{B}+\kappa_{0}, \\
\psi_{\text {approx }}(x) & =\psi_{B}(x)+2 k_{B} \kappa_{0} S_{k_{B}} \psi_{B} .
\end{aligned}
$$

In the next section, we implement this approximation scheme for a specific example.

3.3. An example perturbation calculation. We will use again the piecewise constant potential $V(x)$ in Figure 2.1 as an example. Recall that the bound state eigenfunction is given by

$$
\psi_{B}(x)= \begin{cases}\cos \left(k_{B} x\right) & \text { for } 0 \leq x<a, \\ \cos \left(k_{B} a\right) e^{-\sqrt{V_{0}-k_{B}^{2}}(x-a)} & \text { for } x>a .\end{cases}
$$

To obtain $\kappa_{0}$ in Step 3, we solve for $\phi:=2 k_{B} S\left[k_{B}\right] \psi_{B}$. There are two sections to the potential $V(x)$, and therefore

$$
\begin{aligned}
& -u_{B}^{\prime \prime}-k_{B}^{2} u_{B}=\cos \left(k_{B} x\right), \text { for } 0<x<a, \\
& -u_{B}^{\prime \prime}+\sigma_{B}^{2} u_{B}=\cos \left(k_{B} a\right) e^{-\sqrt{V_{0}-k_{B}^{2}}(x-a)}, \text { for } a<x<L .
\end{aligned}
$$

Hence, we have

$$
u_{B}(x)=-\frac{x \sin \left(k_{B} x\right)}{2 k_{B}}, \text { for } 0 \leq x<a .
$$

Letting $A=-a \sin \left(k_{B} a\right) /\left(2 k_{B}\right)$, and $B=-\left(\sin k_{B} a+a k_{B} \cos \left(k_{B} a\right)\right) /\left(2 k_{B}\right)$, we have, for $a<x<L$

$$
u_{B}(x)=u_{B h}(x)+u_{B p}(x),
$$

where $u_{B h}(x)$ and $u_{B p}(x)$ are the homogeneous and particular solutions, given by

$$
u_{B h}(x)=\frac{1}{2 \sigma_{B}} e^{-\sigma_{B}(x-a)}\left(A \sigma_{B}-B\right)+\frac{1}{2 \sigma_{B}} e^{\sigma_{B}(x-a)}\left(A \sigma_{B}+B\right),
$$

and

$$
u_{B p}(x)=-\left[\left(\frac{1}{4 \sigma_{B}^{2}}+\frac{(x-a)}{2 \sigma_{B}}\right) e^{-\sigma_{B}(x-a)}-\frac{1}{4 \sigma_{B}^{2}} e^{\sigma_{B}(x-a)}\right] \cos \left(k_{B} a\right) .
$$




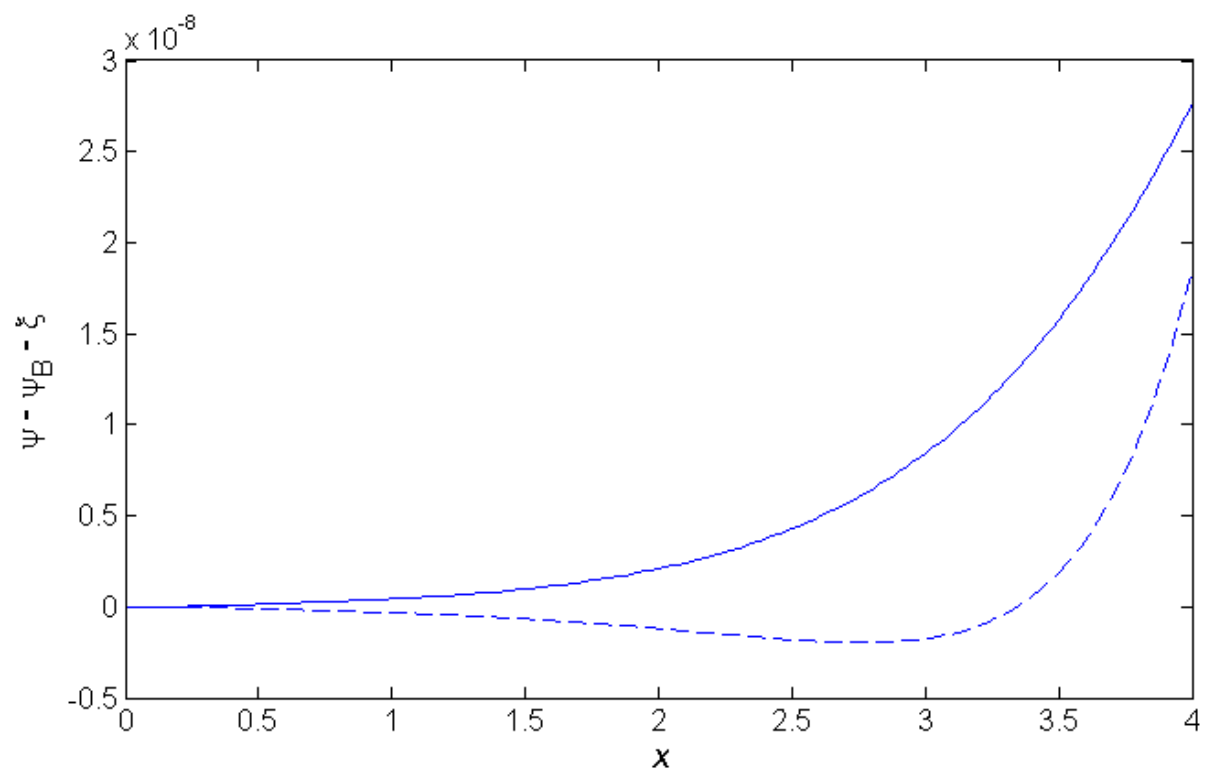

FIG. 3.1. The graph of the approximation error $\psi(x, k)-\psi$ approx $(x)$ when $L=4$. The solid and dashed curves correspond to the real and imaginary parts of the expression.

To obtain the approximation $\kappa_{0}$, one puts $u_{B}=S_{k_{B}} \psi_{B}$ into (3.18):

$$
\kappa_{0}=\frac{\left(i k_{B}+\sigma_{B}\right) \cos \left(k_{B} a\right) e^{-\sigma_{B}(L-a)}}{2 k_{B}\left(u_{B}^{\prime}(L)-i k_{B} u_{B}(L)\right)-i \cos \left(k_{B} a\right) e^{-\sigma_{B}(L-a)}} .
$$

We compare in Table 3.1 the true resonances $k$ with the values $k_{B}+\kappa$ computed by the perturbative approach for different values of $L$. The agreement between the resonance value and the approximated value via the perturbation approach is excellent; the approximation becomes more accurate as $L$ increases.

\begin{tabular}{|c|c|c|}
\hline$L$ & $k$ & $k_{B}+\kappa_{0}$ \\
\hline \hline 4 & $1.029857602509223-0.000016776320240 i$ & $1.029857602963758-0.000016776618066 i$ \\
\hline 5 & $1.029866239929701-0.000000543943637 i$ & $1.029866239930306-0.000000543944139 i$ \\
\hline 6 & $1.029866519939643-0.000000017635724 i$ & $1.029866519939644-0.000000017635725 i$ \\
\hline \multicolumn{2}{|c|}{ TABLE 3.1 } \\
Comparison of the true resonance values $k$ and the approximate value obtained obtained by
\end{tabular}
perturbing the bound-state values $k_{B}$. Note that accuracy increases with barrier thickness $L$.

We assess the accuracy of the approximation to the quasi-normal mode. The agreement between the exact solution $\psi(x, k)$ and $\psi_{\text {approx }}(x)$ for $L=4$ is so good that we cannot visually discern between them. As a result, we plot the real and imaginary parts of $\psi(x, \kappa)-\psi_{\text {approx }}(x)$, which we show in Figure 3.1.

4. High-frequency resonances. Having studied the properties of resonances that are near to bound states, we now turn our attention to the case where the resonance $k$ has a large real part. The energies $k^{2}$ are far away from the bound state energies and lie in the lower half complex plane. 
We will observe that, for large $k$ and $L$, the solution of equations (2.1-2.3) depends negligibly on the function $v(x)$ describing the defect in the interval $[0, a]$. First, let us consider the simplest case in which $V(x) \equiv V_{0}$ in $[0, L]$, a potential barrier of width $L$ without the well. In this case, the solution is $\psi(x)=\cos \sqrt{k^{2}-V_{0}} x$, and the radiation condition $\psi^{\prime}(L)-i k \psi(L)=0$ yields

$$
e^{2 i L \sqrt{k^{2}-V_{0}}}=-\frac{4 k^{2}}{V_{0}}\left(1-\frac{V_{0}}{2 k\left(k+\sqrt{k^{2}-V_{0}}\right)}\right)^{2} .
$$

by direct computation or by putting $\psi(x)=\cos \sqrt{k^{2}-V_{0}} x$ into equation (2.9). Thus

$$
i L \sqrt{k^{2}-V_{0}}=\pi i(m+1 / 2)+\log (k)+\log \left(2 \sqrt{V_{0}}\right)+\log \left(1-\frac{V_{0}}{2 k\left(k+\sqrt{k^{2}-V_{0}}\right)}\right),
$$

in which $m$ is an integer. Using the identity $\sqrt{k^{2}-V_{0}}=k-V_{0} /\left(k+\sqrt{k^{2}-V_{0}}\right)$ and setting

$$
c=-\frac{1}{\pi} \log \left(2 / \sqrt{V_{0}}\right)
$$

we obtain

$$
\begin{aligned}
k & =\frac{\pi(m+1 / 2+i c)}{L}-\frac{i}{L} \log (k)+\frac{V_{0}}{k+\sqrt{k^{2}-V_{0}}}-\frac{i}{L} \log \left(1-\frac{V_{0}}{2 k\left(k+\sqrt{k^{2}-V_{0}}\right)}\right) \\
& =\frac{\pi(m+1 / 2+i c)}{L}-\frac{i}{L} \log (k)+\mathcal{O}\left(|k|^{-1}\right) \quad(m \gg 1) .
\end{aligned}
$$

It seems natural to drop the $\mathcal{O}\left(|k|^{-1}\right)$ term and use the remaining terms as an approximation for an iterative method for computing the resonances,

$$
k \approx \frac{\pi(m+1 / 2+i c)}{L}-\frac{i}{L} \log k .
$$

Then setting

$$
k=\frac{\pi(m+1 / 2+i c)}{L}(1+\kappa)
$$

leads to

$$
\kappa=f_{\mathrm{ap}}(\kappa):=\frac{-i}{\pi(m+1 / 2+i c)}\left(\log \left(\frac{\pi(m+1 / 2+i c)}{L}\right)+\log (1+\kappa)\right)
$$

and hence to the iterative scheme with initial approximation $\kappa_{0}=0$ and recursion rule

$$
\kappa_{n+1}=f_{\text {ap }}\left(\kappa_{n}\right) .
$$

Observe that $f_{\text {ap }}$ does not depend on the defect in the interval $[0, a]$. Numerical calculations with $v(x)=V_{0}$ show that fixed points of $f_{\text {ap }}$ are altered only negligibly if the full formula in (4.2) is used, as demonstrated in Table 4.3. 
If we put $v(x)=0$ in $[0, a], a<L$, corresponding to a square well, the resonance equation becomes

$$
\begin{aligned}
e^{2 i \sqrt{k^{2}-V_{0}}(L-a)} & =-\frac{1+\frac{\sqrt{k^{2}-V_{0}}}{k}}{1-\frac{\sqrt{k^{2}-V_{0}}}{k}} \frac{\cos k a-i \frac{k}{\sqrt{k^{2}-V_{0}}} \sin k a}{\cos k a+i \frac{k}{\sqrt{k^{2}-V_{0}}} \sin k a} \\
& =-\frac{k^{2}}{V_{0}}\left(1+\frac{\sqrt{k^{2}-V_{0}}}{k}\right)^{2} \frac{\cos k a-i \frac{k}{\sqrt{k^{2}-V_{0}}} \sin k a}{\cos k a+i \frac{k}{\sqrt{k^{2}-V_{0}}} \sin k a} \\
& =-\frac{4 k^{2}}{V_{0}}\left(1-\frac{V_{0}}{2 k\left(k+\sqrt{k^{2}-V_{0}}\right)}\right)^{2} \frac{e^{-i k a}-\frac{i V_{0}}{k^{2}-V_{0}+k \sqrt{k^{2}-V_{0}}} \sin k a}{e^{i k a}+\frac{i V_{0}}{k^{2}-V_{0}+k \sqrt{k^{2}-V_{0}}} \sin k a} .
\end{aligned}
$$

Comparing this equation to (4.1), we see that for $\operatorname{Im} k<0$, which is the case for the resonances we seek, there is an additional factor on the left decaying like $e^{-2|\operatorname{Im} k| a}$. This factor is not obviously compensated on the right because of the exponentially increasing behavior of $\sin k a$. When $L$ is not too big, numerical computations show that the approximation (4.3) produces $k$-values whose imaginary parts do in fact deviate appreciably from those of the true resonances. We calculate the exact resonances by a recursion rule $\kappa_{n+1}=f_{\text {ex }}\left(\kappa_{n}\right)$ obtained from (4.7) without making any approximations. As $L$ increases, the approximate results become very good, as demonstrated in Table 4.3. Thus we see that, for small $L$, the imaginary parts of the resonances are sensitive to the form of the well. The error in the imaginary part in that table for $L=4$ even for large $k$ is likely due at least in part to the discontinuity of the square-well potential. A full analysis of the accuracy of the iteration (4.6) is evidently subtle and delicate, and it is not our intention to carry this analysis out here.

Remark 4.1 It is tempting to examine the WKB solution of (2.1)-2.2) with a general potential $v(x)$ for $0<x<a$. We found that such an approach needs to be treated with caution because of the subtlety related to an exponential factor $e^{|\operatorname{Im} k|}$ appearing in the WKB expression. See Appendix F for more details.

Tables 4.1 and 4.2 compare the approximation of $k$ given by (4.4) with $\kappa=0$ to the value obtained by setting $\kappa$ equal to the limit of the sequence $\kappa_{n}$ defined by the iteration (4.6). When $V_{0}=4$, we have $c=0$, and the initial approximation of $k$ is real; this is shown in Table 4.1 . The term $1 / 2+i c$ in $f_{\text {ap }}$ is evidently not unimportant; it turns out that it contributes to a shift in the real parts of the computed resonances that is on the order of the spacing between the resonances $k_{m}$.

Figure 4.1 shows the computed value of $k$ for $V_{0}=4$ and for various values of $m$ and $L$. One observes that the density of resonances increases linearly with the width $L$ of the potential wall, in accordance with theoretical results $[10,4,9]$, and that the resonances lie on a curve in the lower complex half-plane that tends to the real line as $L \rightarrow \infty$, a phenomenon that is also known to occur within the spectral bands of a one-dimensional layered medium as the number of layers increases [6]. 


\begin{tabular}{|c|c|c|c|c|}
\hline$m$ & $k$ & $\pi(m+1 / 2+i c) / L$ & $|\kappa|$ & $|\kappa|$ bound \\
\hline \hline 55 & $43.5842-0.943732 i$ & 43.5896 & 0.0216508 & 0.0432995 \\
\hline 56 & $44.3697-0.948196 i$ & 44.3750 & 0.0213681 & 0.0427344 \\
\hline 57 & $45.1551-0.952582 i$ & 45.1604 & 0.0210936 & 0.0421854 \\
\hline 58 & $45.9406-0.956891 i$ & 45.9458 & 0.0208268 & 0.0416519 \\
\hline 59 & $46.7260-0.961128 i$ & 46.7312 & 0.0205675 & 0.0411333 \\
\hline 60 & $47.5115-0.965295 i$ & 47.5166 & 0.0203152 & 0.0406287 \\
\hline 61 & $48.2970-0.969393 i$ & 48.3020 & 0.0200697 & 0.0401378 \\
\hline 62 & $49.0824-0.973424 i$ & 49.0874 & 0.0198307 & 0.0396599 \\
\hline 63 & $49.8679-0.977392 i$ & 49.8728 & 0.0195980 & 0.0391945 \\
\hline 64 & $50.6533-0.981298 i$ & 50.6582 & 0.0193712 & 0.0387410 \\
\hline 65 & $51.4388-0.985144 i$ & 51.4436 & 0.0191502 & 0.0382991 \\
\hline
\end{tabular}

The values of the quantities in equation (4.4) for barrier width $L=4$ and height $V_{0}=4$, for which $c=0$. The last column is the radius of $D$ in Proposition 4.2.

\begin{tabular}{|c|c|c|c|c|}
\hline$m$ & $k$ & $\pi(m+1 / 2+i c) / L$ & $|\kappa|$ & $|\kappa|$ bound \\
\hline \hline 55 & $29.0569-0.485209 i$ & $29.0597+0.0763576 i$ & 0.0193247 & 0.0386485 \\
\hline 56 & $29.5806-0.488185 i$ & $29.5833+0.0763576 i$ & 0.0190833 & 0.0381657 \\
\hline 57 & $30.1042-0.491109 i$ & $30.1069+0.0763576 i$ & 0.0188485 & 0.0376962 \\
\hline 58 & $30.6278-0.493982 i$ & $30.6305+0.0763576 i$ & 0.0186201 & 0.0372394 \\
\hline 59 & $31.1515-0.496807 i$ & $31.1541+0.0763576 i$ & 0.0183979 & 0.0367949 \\
\hline 60 & $31.6751-0.499585 i$ & $31.6777+0.0763576 i$ & 0.0181814 & 0.0363621 \\
\hline 61 & $32.1987-0.502317 i$ & $32.2013+0.0763576 i$ & 0.0179707 & 0.0359406 \\
\hline 62 & $32.7224-0.505005 i$ & $32.7249+0.0763576 i$ & 0.0177653 & 0.0355298 \\
\hline 63 & $33.2460-0.507651 i$ & $33.2485+0.0763576 i$ & 0.0175651 & 0.0351294 \\
\hline 64 & $33.7696-0.510255 i$ & $33.7721+0.0763576 i$ & 0.0173698 & 0.0347390 \\
\hline 65 & $34.2932-0.512819 i$ & $34.2957+0.0763576 i$ & 0.0171794 & 0.0343582 \\
\hline
\end{tabular}

The values of the quantities in equation (4.4) for barrier width $L=4$ and height $V_{0}=10$, for which $c \neq 0$. The last column is the radius of $D$ in Proposition 4.2.

Proposition 4.2 establishes the convergence of the iteration (4.6) for large enough integers $m$. The following simple estimate is needed in the proof. Let $z_{1}$ and $z_{2}$ be complex numbers. Then

$$
\left|\log \left(1+z_{1}\right)-\log \left(1+z_{2}\right)\right| \leq 4\left|z_{1}-z_{2}\right|, \quad \text { for all } z_{1}, z_{2} \in\left\{|z| \leq \frac{1}{2}\right\} .
$$

\section{Proposition 4.2 If}

$$
\frac{8}{\pi|m+1 / 2+i c|}<1 \quad \text { and } \quad \frac{4}{\pi|m+1 / 2+i c|}\left|\log \frac{\pi(m+1 / 2+i c)}{L}\right|<1,
$$

then $f_{\text {ap }}$ is a contraction of the disk

$$
D=\left\{\kappa:|\kappa|<\frac{2}{\pi|m+1 / 2+i c|}\left|\log \frac{\pi(m+1 / 2+i c)}{L}\right|\right\}
$$

into itself and therefore $D$ contains a unique solution $\kappa$ to (4.5). 


\begin{tabular}{|c|c|c|c|c|}
\hline$m$ & $\pi(m+1 / 2+i c) / L$ & $k_{\text {approx }}$ & $k_{\text {exact }}, v(x)=V_{0}$ & $k_{\text {exact }}, v(x)=0$ \\
\hline \hline \multicolumn{5}{|c|}{$L=4$} \\
\hline 55 & $43.590+0.0279 i$ & $43.585-0.8600 i$ & $43.674-0.8585 i$ & $43.594-0.7153 i$ \\
\hline 120 & $94.641+0.0279 i$ & $94.638-1.1096 i$ & $94.664-1.1094 i$ & $94.581-0.8873 i$ \\
\hline 185 & $145.70+0.0279 i$ & $145.69-1.2175 i$ & $145.71-1.2174 i$ & $145.47-0.9736 i$ \\
\hline 250 & $196.74+0.0279 i$ & $196.74-1.2926 i$ & $196.75-1.2925 i$ & $196.99-1.0343 i$ \\
\hline 315 & $247.79+0.0279 i$ & $247.79-1.3503 i$ & $247.80-1.3502 i$ & $247.88-1.0802 i$ \\
\hline 380 & $298.84+0.0279 i$ & $298.84-1.3971 i$ & $298.85-1.3971 i$ & $298.77-1.1176 i$ \\
\hline \hline \multicolumn{5}{|c|}{$L=50$} \\
\hline 600 & $37.731+0.0022 i$ & $37.731-0.0704 i$ & $37.797-0.0703 i$ & $37.794-0.0689 i$ \\
\hline 1500 & $94.279+0.0022 i$ & $94.279-0.0887 i$ & $94.306-0.0887 i$ & $94.304-0.0869 i$ \\
\hline 2400 & $150.83+0.0022 i$ & $150.83-0.0981 i$ & $150.84-0.0981 i$ & $150.84-0.0962 i$ \\
\hline 3300 & $207.38+0.0022 i$ & $207.38-0.1045 i$ & $207.39-0.1045 i$ & $207.39-0.1024 i$ \\
\hline 4200 & $263.93+0.0022 i$ & $263.93-0.1093 i$ & $263.94-0.1093 i$ & $263.93-0.1071 i$ \\
\hline 5100 & $320.47+0.0022 i$ & $320.47-0.1132 i$ & $320.48-0.1132 i$ & $320.48-0.1109 i$ \\
\hline \hline \multicolumn{5}{|c|}{$L=200$} \\
\hline 2700 & $42.419+0.0006 i$ & $42.419-0.0251 i$ & $42.423-0.0251 i$ & $42.423-0.0246 i$ \\
\hline 6200 & $97.397+0.0006 i$ & $97.397-0.0223 i$ & $97.423-0.0223 i$ & $97.423-0.0222 i$ \\
\hline 9700 & $152.38+0.0006 i$ & $152.38-0.0246 i$ & $152.39-0.0246 i$ & $152.40-0.0244 i$ \\
\hline 13,200 & $207.35+0.0006 i$ & $207.35-0.0261 i$ & $207.37-0.0261 i$ & $207.37-0.0260 i$ \\
\hline 16,700 & $262.33+0.0006 i$ & $262.33-0.0273 i$ & $262.34-0.0273 i$ & $262.35-0.0272 i$ \\
\hline 20,200 & $317.31+0.0006 i$ & $317.31-0.0282 i$ & $317.32-0.0282 i$ & $317.32-0.0281 i$ \\
\hline \multicolumn{5}{|c|}{ TABLE 4.3 } \\
\hline
\end{tabular}

A comparison of computations of resonances $k_{m}$. The first two values are $k_{m}=(\pi(m+1 / 2+$ ic) $/ L)(1+\kappa)$, with $\kappa=0$ and $\kappa=\kappa_{*}$, where $\kappa_{*}=f_{\text {ap }}\left(\kappa_{*}\right)$. The exact resonances $k_{\text {exact }}$ are calculated by a recursion $\kappa_{n+1}=f_{\mathrm{ex}}\left(\kappa_{n}\right)$ obtained from the resonance equations (4.1) for $v(x)=V_{0}$ and (4.7) for $v(x)=0$ without making any approximations. Observe the deviation between the imaginary parts of $k_{\text {approx }}$ and $k_{\text {exact }}$ for $v(x)=0$ for various values of $L$ (barrier width) over comparable ranges of Re $k$. This deviation is significant for small $L$ but diminishes as $L$ increases. The values $a=1$ (well width) and $V_{0}=5$ (barrier height) are used throughout.

Consequently, there is a unique value of $k$ solving (4.3) exactly such that

$$
\left|k-\frac{\pi(m+1 / 2+i c)}{L}\right|<\frac{2}{L}\left|\log \frac{\pi(m+1 / 2+i c)}{L}\right| .
$$

Proof. . To see that $f_{\text {ap }}$ maps $D$ into itself, assume $\kappa \in D$ and use (4.8) and the hypotheses of the theorem to obtain

$$
\begin{array}{r}
\left|f_{\text {ap }}(\kappa)\right| \leq \frac{1}{\pi|m+1 / 2+i c|}\left(\left|\log \frac{\pi(m+1 / 2+i c)}{L}\right|+|\log (1+\kappa)|\right) \\
\leq \frac{1}{\pi|m+1 / 2+i c|}\left(\left|\log \frac{\pi(m+1 / 2+i c)}{L}\right|+4|\kappa|\right) \\
\quad \leq \frac{2}{\pi|m+1 / 2+i c|}\left|\log \frac{\pi(m+1 / 2+i c)}{L}\right|
\end{array}
$$

so that $f_{\text {ap }}(\kappa) \in D$.

To see that $f_{\text {ap }}$ is a contraction in $D$, let $\kappa_{1}$ and $\kappa_{2}$ be in $D$ and use (4.8) to 


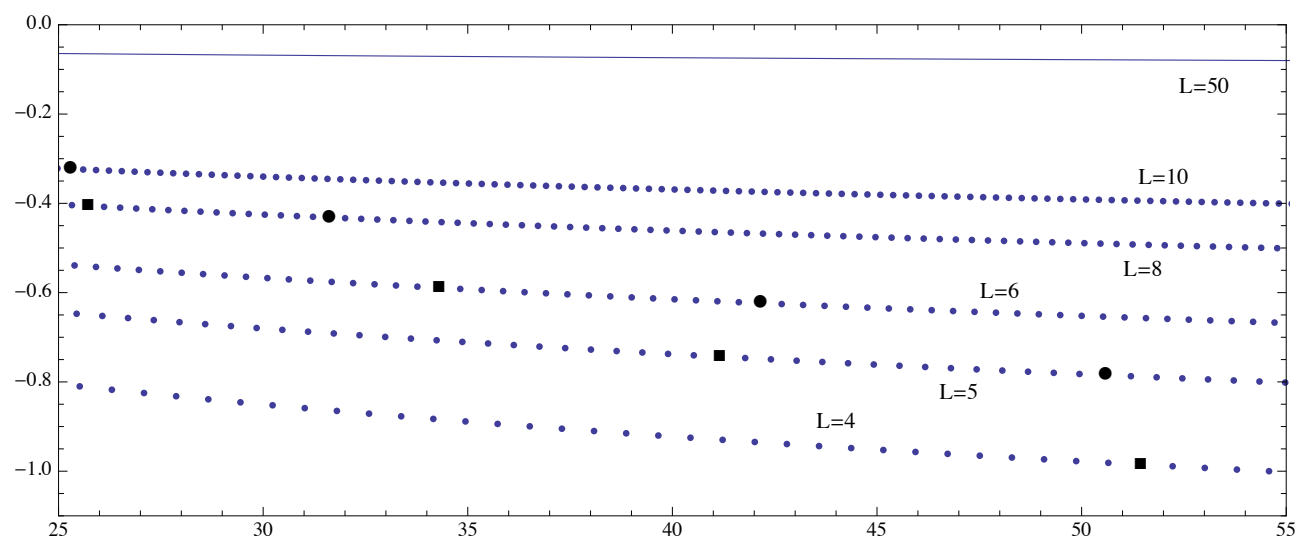

FIG. 4.1. Resonances $k_{m}$ (plotted in the complex plane) computed by the approximate iterative algorithm $(4.4,4.5)$ with barrier height $V_{0}=4$ and for various values of barrier width $L$. The squares show $k_{m}$ for $m=65$, and the circles for $m=80$.

obtain

$$
\begin{aligned}
\left|f_{\text {ap }}\left(\kappa_{1}\right)-f_{\text {ap }}\left(\kappa_{2}\right)\right|=\frac{1}{\pi|m+1 / 2+i c|} & \left|\log \left(1+\kappa_{1}\right)-\log \left(1+\kappa_{2}\right)\right| \\
& \leq \frac{4}{\pi|m+1 / 2+i c|}\left|\kappa_{1}-\kappa_{2}\right| \leq \frac{1}{2}\left|\kappa_{1}-\kappa_{2}\right| .
\end{aligned}
$$

Since the solutions to (4.5), or $f(\kappa)=\kappa$, correspond to the solutions $k$ of the resonance equation (4.3) through the relation (4.4), the unique solution of $f(\kappa)=\kappa$ in $D$ yields a unique resonant value $k$ satisfying the inequality claimed in the last statement of the theorem.

5. Discussions. Motivated by the desire to devise a computational method for fast and accurate calculation of resonances, we studied the properties of the resonances of a Schrödinger's equation with a potential well that has thick barriers. We showed that the resonances associated with such a potential are close to the defect mode eigen-frequencies of the limiting potential in which the barrier thickness goes to infinity. We established that the difference between the eigen-frequencies and the nearby resonances is exponentially small in the barrier thickness. Inspired by this finding, we developed a perturbational method for approximating resonances that are simple to implement and accurate. To complete our study, we explored the highfrequency resonances of potentials with thick barriers and obtained a picture of their behavior as the barrier thickness becomes large.

The method developed here takes advantage of the one-dimensional nature of the problem. It relies on the theory of ordinary differential equations - the structure of solutions, the role of the Wronskian, existence and uniqueness of the initial-value problem, exact solutions in the barrier, and an exact description of the outgoing condition. We expect the techniques to be applicable to higher-dimensional problems with radial symmetry. It is not clear how this work can be extended to treat general higher-dimensional problems.

A natural extension of the present work is to the study of resonances of a onedimensional finite (truncated) periodic structure with a defect and to compare them to localized defect modes of its infinite periodic counterpart. One simply replaces 
the uniform barrier with a periodic one. By the Floquet theory, the solutions of the time-independent Schrödinger equation in the barrier are periodically distorted exponential functions. Thus, if one takes the end $L$ of the barrier to tend to infinity through a sequence $L=n d$, where $d$ is the period and $n$ is an integer, the calculations in this paper remain essentially unchanged. Now, instead of having bound states only below the bottom of the continuous spectrum of the infinite-barrier problem, there may be defect modes within the gaps of the spectrum created by the periodicity of the potential. Preliminary results for the case of a piecewise constant medium show that the difference between the frequencies of the defect mode of the infinite periodic medium and the resonances of its finite counterpart is exponentially small in the number of periodic layers.

Acknowledgements. The authors wish to thank the Banff International Research Station for its hospitality. This research was initiated during the authors' participation in the BIRS "Research in Teams" event "Research in photonics: modeling, analysis, and optimization" in September of 2010. This research was also supported in part by grants NSF DMS-0537015 (DCD), NSF DMS-0807856 (FS), NSF DMS-0807325 (SPS) and NSF DMS-1008855 (MIW).

\section{REFERENCES}

[1] David Bindel and Maciej Zworski, Theory and Computation of Resonances in $1 D$ Scattering, URL: http://www.cims.nyu.edu/ dbindel/resonant1d/ (2006).

[2] Anne-Sophie Bonnet-BenDhia and Felipe Starling, Guided waves by electromagnetic gratings and nonuniqueness examples for the diffraction problem, Math. Meth. Appl. Sci., Vol 17, No. 5, 305-338 (1994).

[3] Kiril Datchev, Computing Resonances by Generalized Complex Scaling, Preprint. URL: http://math.mit.edu/ datchev/res.ps (2006).

[4] Richard Froese, Asymptotic Distribution of Resonances in One Dimension, J. Differ. Equations, Vol. 137, 251-272 (1997).

[5] B. Helffer and J. Sjöstrand, Résonances en limite semi-classique, Mémoires de la Soc. Math. de Fr. $2^{e}$ série, Vol. 24-25, (1986), 228 pages.

[6] Alexei Iantchenko, Resonance spectrum for one-dimensional layered media, Applicable Analysis, Vol. 85, No. 11, 1383-1410 (2006).

[7] T. Regge, Analytic Properties of the Scattering Matrix, Il Nuovo Cimento, Vol. 8, No. 5, 671679 (1958).

[8] Barry Simon, The Definition of Molecular Resonance Curves by the Method of Exterior Complex Scaling Phys. Lett., Vol. 71A, No. 2-3, 211-214 (1979).

[9] Barry Simon, Resonances in one dimension and Fredholm determinants, J. Funct. Anal., Vol. $178,396-420$ (2000).

[10] Johannes Sjöstrand and Maciej Zworski, Complex scaling and the distribution of scattering poles, J. AMS, Vol. 4, No. 4, 729-769 (1991).

[11] Musheng Wei, George Majda, and Walter Strauss, Numerical Computation of the Scattering Frequencies for Acoustic Wave Equations, J. Comp. Phys., Vol. 75, 345-358 (1988).

[12] Maciej Zworski, Distribution of Poles for Scattering on the Real Line, J. Functional Analysis, Vol 73, No. 2, 277-296 (1987).

[13] Maciej Zworski, Lectures on Scattering Resonances, Lecture notes, Dept. of Math., UC Berkeley, Preprint. URL: http://math.berkeley.edu/ zworski/res.pdf (2011).

Appendix A. $H_{L}$ has no bound states.

If $\psi$ satisfies $-\psi^{\prime \prime}+V(x) \psi=k^{2} \psi$ and $\psi$ decays to 0 as $x \rightarrow \infty$, then $k^{2}=-\eta^{2}$ for some $\eta>0$. Multiplying the differential equation by $\bar{\psi}$ and integrating over the interval $[a, b]$ yields

$$
\int_{a}^{b}\left(V(x)+\eta^{2}\right)|\psi|^{2}=\int_{a}^{b} \psi^{\prime \prime} \bar{\psi}=-\int_{a}^{b}\left|\psi^{\prime}\right|^{2}+\psi^{\prime}(b) \bar{\psi}(b)-\psi^{\prime}(a) \bar{\psi}(a) .
$$


Under the assumption that $V(x) \geq 0$, taking $a \rightarrow \infty$ and $b \rightarrow-\infty$ (full-line problem) or $a \rightarrow \infty$ and $b=0$ (half-line problem) causes the terms of evaluation at $a$ and $b$ to vanish, leaving $\psi(x) \equiv 0$.

\section{Appendix B. Resonances are in the lower half plane.}

Let $\psi$ satisfy equations $(2.1-2.3)$, with Re $k>0$. Multiplying the ODE by the complex conjugate $\bar{\psi}(x)$ and integrating by parts from 0 to $x$ yields

$$
\int_{0}^{x}\left|\psi^{\prime}(y)\right|^{2} d y-\psi^{\prime}(x) \bar{\psi}(x)+\int_{0}^{x} V(y)|\psi(y)|^{2} d y-k^{2} \int_{0}^{x}|\psi(y)|^{2} d y=0 .
$$

Using $\psi(x)=C e^{i k x}$ for $x>L$ from the outgoing condition (2.3), the imaginary part of the later equation is

$$
\operatorname{Re} k|C|^{2} e^{-2 \operatorname{Im}(k) x}+\operatorname{Im}\left(k^{2}\right) \int_{0}^{x}|\psi|^{2}=0 .
$$

Recalling that $\operatorname{Re} k>0$, we see that $\mathrm{k}=0 \Longrightarrow C=0 \Longrightarrow \psi=0$. If $\operatorname{Im} k>0$, then the left-hand term tends to zero as $x \rightarrow \infty$, which implies that $\int_{0}^{\infty}|\psi|^{2}=0$, so that $\psi=0$. We conclude that $\operatorname{Im} k<0$.

\section{Appendix C. Point spectrum is finite.}

We give a proof of the well-known fact that the point spectrum for $H_{\infty}(x)$ is finite. The proof is adapted from [2, Thm. 3.3] and quantifies the number of bound states as the number of Neumann eigenvalues of the well that are less than $V_{0}$.

The self-adjoint operator $-\partial_{x}^{2}+V(x)$ on $(0, a)$ with homogeneous boundary conditions $\psi^{\prime}(0)=0$ and $\psi^{\prime}(a)+\sqrt{V_{0}-k^{2}} \psi(a)=0$ has form domain equal to $H^{1}(a, b)$ on which its associated positive form is given by

$$
A_{k}(\psi, \phi):=\int_{0}^{a}\left(\psi^{\prime} \bar{\phi}^{\prime}+V(x) \psi \bar{\phi}\right) d x+\sqrt{V_{0}-k^{2}} \psi(a) \bar{\phi}(a) .
$$

A bound-state pair $\left(k_{B}, \psi_{B}\right)$ is characterized by the equation

$$
A_{k_{B}}\left(\psi_{B}, \phi\right)=k_{B}^{2} \int_{0}^{a} \psi_{B} \bar{\phi} d x \quad \forall \phi \in H^{1}(0, a) .
$$

The form $A_{k}$ admits a sequence of eigenfunctions $\psi_{j}(k ; x)$ associated with eigenvalues $\left\{\lambda_{j}(k)\right\}_{j=1}^{\infty}$ that tends to infinity, defined by

$$
A_{k}\left(\psi_{j}(k), \phi\right)=\lambda_{j}(k) \int_{0}^{a} \psi \bar{\phi} d x \quad \forall \phi \in H^{1}(0, a) .
$$

One shows that each function $\psi_{j}(k ; x)$ is a decreasing continuous function of $k$ by examining its characterization by means of the Rayleigh quotient,

$$
\lambda_{j}(k)=\min _{X_{j} \in S_{j}\left(H^{1}\right)} \max _{\substack{\psi \in X_{j} \\ \psi \neq 0}} \frac{A_{k}(\psi, \psi)}{\int_{0}^{a} \psi^{2} d x},
$$

in which $S_{j}\left(H^{1}\right)$ denotes the set of $j$-dimensional subspaces of $H^{1}$. Now, each boundstate value $k_{B}$ satisfies, for some $j$,

$$
k_{B}^{2}=\lambda_{j}\left(k_{B}\right)
$$


Since the $\lambda_{j}(k)$ are decreasing and continuous, the number of such relations for $k<$ $\sqrt{V_{0}}$ is equal to the number of eigenvalues of $A_{\sqrt{V_{0}}}$ that are less than $V_{0}$. Since the boundary term in $A_{\sqrt{V_{0}}}$ vanishes, this form is the Neumann one. Thus the number of bound states of $V(x)$ is equal to the number of Neumann eigenvalues of $-\partial_{x}^{2}+V(x)$ on $(0, a)$ that are less than $V_{0}$, and this is finite.

Appendix D. Eigenvalues of $H_{\infty}$, zeros of $F_{B}(k)$, are simple.

We differentiate the expression $i F_{B}(z)$, which is real for $k^{2}<V_{0}$ :

$$
\frac{d}{d k}\left(i F_{B}(k)\right)=-\frac{d}{d k}\left[\sqrt{V_{0}-k^{2}}+\left(\frac{\psi^{\prime}(a, k)}{\psi(a, k)}\right)\right]
$$

Note that

$$
\frac{\partial}{\partial k}\left(\frac{\psi^{\prime}(a, k)}{\psi(a, k)}\right)=\frac{\dot{\psi}^{\prime}(a, k) \psi(a, k)-\psi^{\prime}(a, k) \dot{\psi}(a, k)}{\psi(a, k)^{2}}
$$

where $\frac{\partial \psi}{\partial k}=\dot{\psi}$, and similarly for $\frac{\partial \psi^{\prime}}{\partial k}=\dot{\psi}^{\prime}$. Differentiating (2.1), we find that $\dot{\psi}$ satisfies

$$
\begin{aligned}
-\dot{\psi}^{\prime \prime}+V(x) \dot{\psi} & =k^{2} \dot{\psi}+2 k \psi \quad \text { in }(0, a), \\
\dot{\psi}(0, k) & =\dot{\psi}^{\prime}(0, k)=0,
\end{aligned}
$$

where $\psi$ solves (2.1). Multiplying (D.2) by $\psi$ and integrating by parts, we find

$$
\dot{\psi}^{\prime}(a, k) \psi(a, k)=\int_{0}^{a} \dot{\psi}^{\prime} \psi^{\prime}+\left(V(x)-k^{2}\right) \dot{\psi} \psi-2 k \psi^{2} d x .
$$

On the other hand, multiplying (2.1) by $\dot{\psi}$ and integrating by parts,

$$
\psi^{\prime}(a, k) \dot{\psi}(a, k)=\int_{0}^{a} \psi^{\prime} \dot{\psi}^{\prime}+\left(V(x)-k^{2}\right) \psi \dot{\psi} d x
$$

Combining these expressions, we obtain

$$
\frac{\partial}{\partial k}\left(\frac{\psi^{\prime}(a, k)}{\psi(a, k)}\right)=\frac{-2 k \int_{0}^{a} \psi^{2} d x}{\psi^{2}(a, k)} .
$$

Note that when $k^{2}<V_{0}$, all parameters in (2.1) are real, and the solution $\psi(x, k)$ is hence real. Further, because $\psi(0, k)=1$ and $\psi$ is continuous, we conclude $\int_{0}^{a} \psi(x, k)^{2} d x>$ 0 . Finally, we obtain

$$
\frac{d}{d k}\left(i F_{B}(k)\right)=k\left(\frac{1}{\sqrt{V_{0}-k^{2}}}+\frac{2 \int_{0}^{a} \psi^{2}(x ; k) d x}{\psi^{2}(a, k)}\right) \neq 0 .
$$

The factor of $k$ is harmless because it disappears when taking the derivative with respect to the eigenvalue $E=k^{2}$ instead of with respect to $k$. (We have assumed that $v(x)>0$ so that $k_{B}>0$, although this is not necessary here.) Since $\psi\left(x, k_{B}\right)$ is exponentially decaying for $x>a, \psi\left(a, k_{B}\right) \neq 0$, and we conclude that

$$
\frac{d}{d k}\left(i F_{B}\left(k_{B}\right)\right)>0
$$


at each eigenvalue $k_{B}$.

Appendix E. Proof that $C_{1} \neq 0$.

Let $\psi_{1}$ and $\psi_{2}$ satisfy $-\psi^{\prime \prime}+V(x) \psi-k^{2} \psi=0$ with

$$
\left[\begin{array}{ll}
\psi_{1}(0) & \psi_{2}(0) \\
\psi_{1}^{\prime}(0) & \psi_{2}^{\prime}(0)
\end{array}\right]=\left[\begin{array}{ll}
1 & 0 \\
0 & 1
\end{array}\right],
$$

so that $W\left[\psi_{1}, \psi_{2}\right](x)=\psi_{1}(x) \psi_{2}^{\prime}(x)-\psi_{2}(x) \psi_{1}^{\prime}(x) \equiv 1$.

When the solution $\psi_{1}$ satisfies the bound-state condition at $x=a$, it is equal to the bound state $\psi_{B}$ in $[0, a]$ :

$$
\psi_{B}^{\prime}(a)+\sigma \psi_{B}(a)=0 \quad\left(\psi_{B}=\psi_{1}\right)
$$

Consider the initial-value problem

$$
\left\{\begin{array}{l}
-u^{\prime \prime}+V(x) u-k^{2} u=\psi_{B}(x), \\
u(0)=u^{\prime}(0)=0 .
\end{array}\right.
$$

By the standard method of variation of parameters, the solution can be written as a variable-coefficient combination of $\psi_{B}$ and $\psi_{2}$ chosen such that

$$
\left\{\begin{array}{l}
u(x)=\gamma_{1}(x) \psi_{B}(x)+\gamma_{2}(x) \psi_{2}(x), \\
u^{\prime}(x)=\gamma_{1}(x) \psi_{B}^{\prime}(x)+\gamma_{2}(x) \psi_{2}^{\prime}(x),
\end{array}\right.
$$

in which

$$
\gamma_{1}(x)=\int_{0}^{x} \psi_{B}(y) \psi_{2}(y) d y, \quad \gamma_{2}(x)=-\int_{0}^{x} \psi_{B}(y)^{2} d y .
$$

For $x \geq a$, the bound state (for $L=\infty$ ) is $\psi_{B}(x)=\psi_{B}(a) e^{-\sigma(x-a)}$, and the equation $-u^{\prime \prime}+V(x) u-k^{2} u=\psi_{B}(x)$ becomes

$$
-u^{\prime \prime}+\sigma^{2} u=\psi_{B}(a) e^{-\sigma(x-a)} .
$$

The general solution of this equation is

$$
u(x)=C_{1} e^{\sigma(x-a)}+\left(C_{2}+\frac{\psi_{B}(a)}{2 \sigma} x\right) e^{-\sigma(x-a)}
$$

Let us obtain $C_{1}$ in terms of $u(a)$ and $u^{\prime}(a)$ :

$$
\begin{aligned}
& u(a)=C_{1}+C_{2}+\frac{\psi_{B}(a)}{2 \sigma} a, \\
& u^{\prime}(a)=\sigma C_{1}-\sigma C_{2}+\frac{\psi_{B}(a)}{2 \sigma}(1-\sigma a),
\end{aligned}
$$

which yields

$$
2 \sigma C_{1}=u^{\prime}(a)+\sigma u(a)-\frac{\psi_{B}(a)}{2 \sigma}
$$


We will show that this is nonzero. Using (E.1) together with the bound-state condition $\psi_{B}^{\prime}(a)+\sigma \psi_{B}(a)=0$, we obtain

$$
2 \sigma C_{1}=\gamma_{2}(a)\left(\psi_{2}^{\prime}(a)+\sigma \psi_{2}(a)\right)-\frac{\psi_{B}(a)}{2 \sigma} .
$$

The condition $C_{1}=0$ together with the bound-state condition give the pair

$$
\left\{\begin{array}{l}
\gamma_{2}(a)\left(\psi_{2}^{\prime}(a)+\sigma \psi_{2}(a)\right)-\frac{\psi_{B}(a)}{2 \sigma}=0 \\
\psi_{B}^{\prime}(a)+\sigma \psi_{B}(a)=0
\end{array}\right.
$$

Multiplying the first of these equations by $\psi_{B}(a)$ and the second by $\gamma_{2}(a) \psi_{2}(a)$ and subtracting yields

$$
\gamma_{2}(a)\left(\psi_{B}(a) \psi_{2}^{\prime}(a)-\psi_{2}(a) \psi_{B}^{\prime}(a)\right)-\frac{\psi_{B}^{2}(a)}{2 \sigma}=0 .
$$

Using the fact that the $W\left[\psi_{B}, \psi_{2}\right](x) \equiv 1$ and the expression (E.2) for $\gamma_{2}$, this equality becomes

$$
-\int_{0}^{a} \psi_{B}(y)^{2} d y-\frac{\psi_{B}^{2}(a)}{2 \sigma}=0,
$$

which is untenable. This means that $C_{1}$ must be nonzero.

Appendix F. WKB analysis.

The formal WKB solution of the initial-value problem $(2.1,2.2)$ is

$$
\psi_{\mathrm{wKB}}(x)=\cos \left(k x-\frac{1}{2 k} \int_{0}^{x} V(y) d y\right)+\mathcal{O}\left(|k|^{-2}\right) e^{|\operatorname{Im} k| x},
$$

in which the $\mathcal{O}\left(|k|^{-2}\right)$ factor is uniform in $x$. This can be obtained rigorously as follows. The solution $\psi$ satisfies the integral equation $\psi=\cos (k \cdot)+k^{-1} T \psi$, where the operator $T$ is defined by

$$
(T \phi)(x):=\int_{0}^{x} V(y) \phi(y) \sin (k(x-y)) d y .
$$

This operator is bounded in the weighted uniform norm $\|\phi\|_{|\operatorname{Im} k|}=\sup _{0<x<L}\left|\phi(x) e^{-|\operatorname{Im} k| x}\right|$. Thus, for large enough $k$, we have an explicit expansion of the solution,

$$
\psi=\left(1+k^{-1} T+k^{-2} T^{2}+\cdots\right) \cos (k \cdot),
$$

which yields $\psi=\psi_{\text {wкв }}$.

The radiation condition (2.3) applied to the WKB solution,

$$
i k \cos \left(k x-\frac{1}{2 k} \int_{0}^{x} V(y) d y\right)+\left(k-\frac{V_{0}}{2 k}\right) \sin \left(k x-\frac{1}{2 k} \int_{0}^{x} V(y) d y\right)=0,
$$

yields the resonance equation

$$
\exp \left(2 i k L-\frac{i}{k} \int_{0}^{L} V(y) d y\right)=1-\frac{4 k^{2}}{V_{0}} .
$$


Excluding a term of order $\mathcal{O}\left(|k|^{-2}\right)$ that comes from the constant "1", we obtain

$$
k=\frac{\pi(m+1 / 2+i c)}{L}-\frac{i}{L} \log k+\frac{1}{2 k L} \int_{0}^{L} V(y) d y .
$$

Observe that, if $V(x) \equiv V_{0}$, then equation (4.2) is recovered up to order $\mathcal{O}\left(|k|^{-1}\right)$. As we have learned from the case $V(x)=0$ for $0<x<a$, in which solutions of this equation approximate the resonances well only for large enough $L$, the WKB result should be treated with caution. This subtlety comes from the exponential factor $e^{|\operatorname{Im} k| x}$ in the asymptotics. 